\title{
Decreased visual performance resulting from temporal uncertainty, target movement, and background movement
}

\author{
KENNETH W. GISH, JAMES B. SHEEHY, and H. W. LEIBOWITZ \\ The Pennsylvania State University, University Park, Pennsylvania
}

\begin{abstract}
Temporal uncertainty, target movement, and background movement degrade contrast sensitivity, grating acuity, and reaction time. The results are interpreted on the basis of fixational instability, processing efficiency, and alertness. Alternative explanations based on masking are also considered. The variables used in this study may be predictive of performance outside the laboratory, inasmuch as they simulate conditions frequently associated with demanding visual tasks encountered in nonlaboratory environments.
\end{abstract}

Visual tests are frequently used to predict performance outside of the laboratory. However, the predictive validity of these tests is limited by the extent to which conditions in the nonlaboratory environment can be simulated in the laboratory. For example, a pilot is typically exposed to high levels of spatial, temporal, and stimulus uncertainty, as well as object- and self-motion. If these variables degrade performance, and are not included in the test procedures, the test results may not accurately predict performance in the natural setting.

Two factors present in many demanding visual tasks are temporal uncertainty and background movement. Temporal uncertainty, which refers to how predictable a stimulus is in time, affects both detection and reaction time. Increasing temporal uncertainty decreases the slope of the receiver operator characteristic, decreases target detectability $\left(d^{\prime}\right)$ (Cohn, 1981; Earle \& Lowe, 1971; Lasley \& Cohn, 1981), and increases reaction time (Wertheim, 1980).

Measurements of acuity and contrast sensitivity are typically obtained with warning signals to reduce temporal uncertainty. This is acceptable if the objective is to determine the physical limits of performance (Geisler, 1984; Geisler \& Davila, 1985); however, if the objective is to predict performance outside the laboratory, then some degree of uncertainty should be incorporated into the test procedures. Surprisingly, the effects of temporal uncertainty on acuity and contrast sensitivity have not been documented.

Several experiments have investigated the effects of background movement on visual performance. MacKay

\footnotetext{
This research was supported by Grant EY03276 from the National Eye Institute and Grant N62269-85-R-0285 from the Office of Naval Research. We are grateful to Gordon Shulman and Naoyuki Osaka for comments on earlier versions of this manuscript. Address requests for reprints to H. W. Leibowitz, Department of Psychology, Moore Bldg., Pennsylvania State University, University Park, PA 16802. K. W. Gish and J. B. Sheehy's present address is CODE 6023, Naval Air Development Center, Warminster, PA 18974.
}

(1970a, 1970b) found a decrease in frequency of seeing a test spot when the spot was presented $20-40 \mathrm{msec}$ before the onset of background movement. Using a squarewave background stimulus, Breitmeyer and Valberg (1979) and Breitmeyer, Valberg, Kurtenbach, and Neumeyer (1980) found decreased ratios of stationary-tomoving thresholds for spot stimuli, with the largest decrease occurring for backgrounds oscillated at $4 \mathrm{~Hz}$.

Recent studies in which the spatial frequency of the background (Bowling, 1985b) or central grating (Bowling, 1985a; Derrington, 1984) was varied showed the largest increases in threshold (or decreases in contrast sensitivity) when the spatial frequencies of the central and background gratings were similar. Also, when the peripheral and central gratings had the same orientation, contrast thresholds for the central gratings were elevated compared with thresholds for different central and peripheral grating orientations (Bowling, 1985a). It is important to note, however, that there was a decrement in contrast sensitivity for both same- and different-orientation central and surround gratings when compared with thresholds obtained with a steady surround. This, in conjunction with the results of Bowling (1985b) and Derrington (1984), suggests that the decrement in performance on grating detection tasks in the presence of background movement is both orientation and spatial-frequency selective.

Moving backgrounds induce involuntary eye movements even when an adequate stimulus for fixation is present. Gippenreiter and Romanov (1974) reported that in the presence of moving backgrounds, subjects instructed to fixate stationary test stimuli showed involuntary nystagmus movements with amplitudes of up to 20' of arc. Such eye movements, termed fixational optokinetic nystagmus (FOKN), could degrade performance on tasks requiring fixational stability. They also showed that more demanding foveal visual tasks reduced but did not eliminate FOKN. This is consistent with a study by Cheng and Outerbridge (1975), who reported a decrease in OKN 
when subjects attended to a blank rectangular central area subtending $30^{\circ}$ horizontally (no fixation stimulus was provided). These results suggest that fixation stability in the presence of background motion is influenced by selective attention. Thus, variables that determine the efficiency of selective attention should affect fixational stability and produce changes in visual performance.

The possibility that background movement interacts with warning condition was investigated in the following series of experiments by manipulating both background motion and temporal uncertainty factorially. If fixational stability is dependent on the efficiency of selective attention and the visual task requires fixational stability for optimal performance, then the presence of a warning signal should reduce degradation due to background motion.

\section{EXPERIMENT 1}

Experiment 1 determined the effect of uncertainty and/or background motion on contrast sensitivity. Derrington (1984) reported that the contrast sensitivity for a 40-msec exposure duration was selectively reduced for spatial frequencies of less than 1.0 cycles per degree (cpd) in the presence of a .2-cpd grating pattern shifted $1 / 2$ cycle in $8 \mathrm{msec}$. Bowling (1985a, 1985b) reported similar decreases in contrast sensitivity for low spatial frequencies in the presence of drifting or flickering backgrounds, suggesting that spatial-frequency-selective masking is involved.

Using a radial grating stimulus rotated about a central $2^{\circ}$ target, Marrocco, Carpenter, and Wright (1985) found a decrement in contrast sensitivity when measured in the presence of background movement. This decrement was observed for dichoptic viewing $\left(2^{\circ}\right.$ target to left eye and radial grating to the right) and in 2 out of 3 subjects for monocular viewing. The monocular background effect was attributed to the periphery (Mcllwain, 1964, 1966) or “jerk" effect (Valberg \& Breitmeyer, 1980), since a background pattern similar to that used to demonstrate the jerk effect (4-Hz square-wave modulation of a squarewave grating) did not affect sensitivity under dichoptic viewing. In the dichoptic viewing condition there was some evidence of spatial frequency selectivity, since there was a monotonic decline in the effect of the background as spatial frequency increased. These results suggest that decrements may be due to interference at early or late visual processing stages.

If fixational instability is induced by background movement, then contrast sensitivity for high spatial frequencies should decrease more in the presence of background movement than should contrast sensitivity for low spatial frequencies (Kelly, 1979; Sheehy, 1984). Alternatively, if the moving background is a low spatial frequency, spatial-frequency-selective masking predicts a larger decrease in contrast sensitivity for the low spatial frequencies (Derrington, 1984; Marrocco et al., 1985). The following experiment was designed to test these predictions.
Temporal uncertainty was introduced by eliminating all cues about the temporal locus of grating presentations. Reaction times for detection responses were also recorded to determine how processing efficiency was affected under each of the conditions in the experiment.

\section{Method}

Subjects. Six paid subjects, each with 20/20 vision or better as determined by a Titmus vision tester, were tested.

Apparatus. Subjects viewed a sine-wave grating displayed on the face of a Tektronix 608 monitor (mean luminance $4.51 \mathrm{fL}$ ) at a distance of $47.5 \mathrm{~cm}$. The pattern, created using $z$-axis modulation (Campbell \& Green, 1965 ), subtended $3.68^{\circ}$ vertically and $5.8^{\circ}$ horizontally.' Contrast (defined as $\left.\left[L_{\max }-L_{\min }\right] /\left[L_{\max }+L_{\min }\right]\right)$, spatial frequency and duration of the $z$-axis were under computer control (D/A channels from an Apple Ile) via voltage-controlled amplitude and frequency inputs on a Wavetek 193 function generator. Gratings were presented for $200 \mathrm{msec}$. A dark circular fixation point (15' of arc) was provided.

The hemicylindrical background subtended $94^{\circ}$ vertically $\times 180^{\circ}$ horizontally at a viewing distance of $47 \mathrm{~cm}$. A shadow-casted square-wave grating $(.16 \mathrm{cpd}, 42 \%$ contrast, mean luminance $1.48 \mathrm{fL}$ ) was created by light from a tubular bulb (3-in. vertical filament) through graphic arts tape ( $1 / \mathrm{s}$ in. wide) spaced evenly on the outer surface of a glass cylinder ( $9.5 \mathrm{in.}$ long, $5.88 \mathrm{in}$. diameter). The glass cylinder was rotated in one direction (left to right) at a constant velocity ( 5 degrees per second [dps] or .8 cycles per second [cps]) about the stationary light source to produce the moving shadows. Crossed polaroids were used to prevent the light from the background and central patterns from interacting. A 100-msec $1-\mathrm{kHz}$ pure tone (72 $\mathrm{dBA}$ ) served as a warning signal in the certainty condition.

Two points should be made about the apparatus. First, there was a . $5-\mathrm{cm}$ (1\% of the viewing distance) difference in distance between the central and background patterns, due mainly to the thickness of the glass on the monitor for the central pattern. Although this difference may have elevated thresholds, this elevation should be constant across conditions, since the fixation spot was always at the same distance. Second, one criterion used in choosing the background spatial frequency was that it be different from the spatial frequency of the targets. This was done to reduce the possible contribution of spatial-frequency-selective masking, since we were more interested in the effects of fixational instability.

Procedure. Each subject was tested under two conditions of certainty (with or without a warning signal) and two background conditions (moving or stationary) per session. Each combination of certainty and background was repeated twice for each subject for a given session. In all, there were eight blocks of trials per session. The ordering of these blocks was counterbalanced among subjects.

There were three sessions in all. The spatial frequency was either 1,4 , or $10 \mathrm{cpd}$ for a given session. Spatial frequency presentation order was randomly determined. The order of background and uncertainty conditions was counterbalanced among subjects.

The subject's task was to press a key when the grating pattern was detected. The subjects were asked to respond as quickly and accurately (that is, to avoid responding to blank trials) as possible to the detected pattern and to maintain fixation on the central fixation spot.

Each block of trials began after a 2-min adaptation period, followed by an up-down staircase procedure used to estimate threshold contrast (mean of six yes/no transitions). These thresholds are not reported, as their purpose was to give the subjects practice before beginning the method-of-constant-stimuli procedure and to provide a rough estimate of threshold to allow a choice of contrast levels that would incorporate the threshold. 
Three contrast levels above and below the staircase threshold, spaced at intervals of .1 log unit, were then used in a method-ofconstant-stimuli procedure. Each of the seven levels was presented in random order 10 times within a block. Ten of the trials were catch trials. In all there were 80 trials per block. Thresholds were defined as the contrast that yielded $75 \%$ detection. This value was determined from the regression equation fit to the linearized psychometric function obtained with the constant-stimuli procedure.

Reaction times for each subject were calculated from the contrast that produced $100 \%$ detections. If no contrast was detected $100 \%$ of the time, reaction times were calculated from the contrast that yielded $90 \%$ detections.

\section{Results}

The mean contrast sensitivity as a function of spatial frequency for the two background types is plotted in Figure 1. Contrast sensitivity was highest at $1 \mathrm{cpd}$ and decreased with spatial frequency. The presence of a warning signal increased sensitivity for all spatial frequencies.

The contrast sensitivity data were log transformed and a $2 \times 2 \times 2 \times 3$ repeated measures analysis of variance (ANOVA) was performed with measurement time (two measurements per condition per subject), warning, background, and spatial frequency as factors. There were significant main effects of spatial frequency $[F(2,10)=$ $28.61, p<.01]$ and warning $[F(1,5)=13.00, p<.02]$. The background degraded performance when there was no warning signal, as indicated by the significant interaction of warning with background $[F(1,5)=20.83$, $p<.01]$.

Figure 2 presents reaction time as a function of spatial frequency. The increase in reaction time with spatial frequency $[F(2,10)=25.31, p<.01]$ and the decrease in reaction time in the presence of a warning signal $[F(1,5)$ $=79.67, p<.01]$ are both significant.

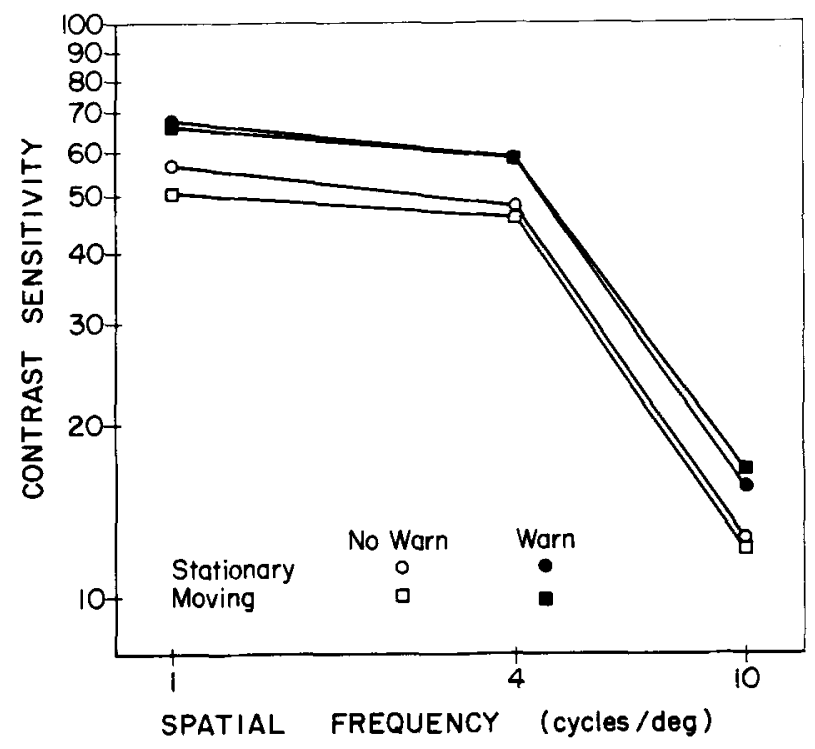

Figure 1. Contrast sensitivity as a function of spatial frequency with and without warning for stationary and drifting $\left(5^{\circ}\right.$ per second) backgrounds.

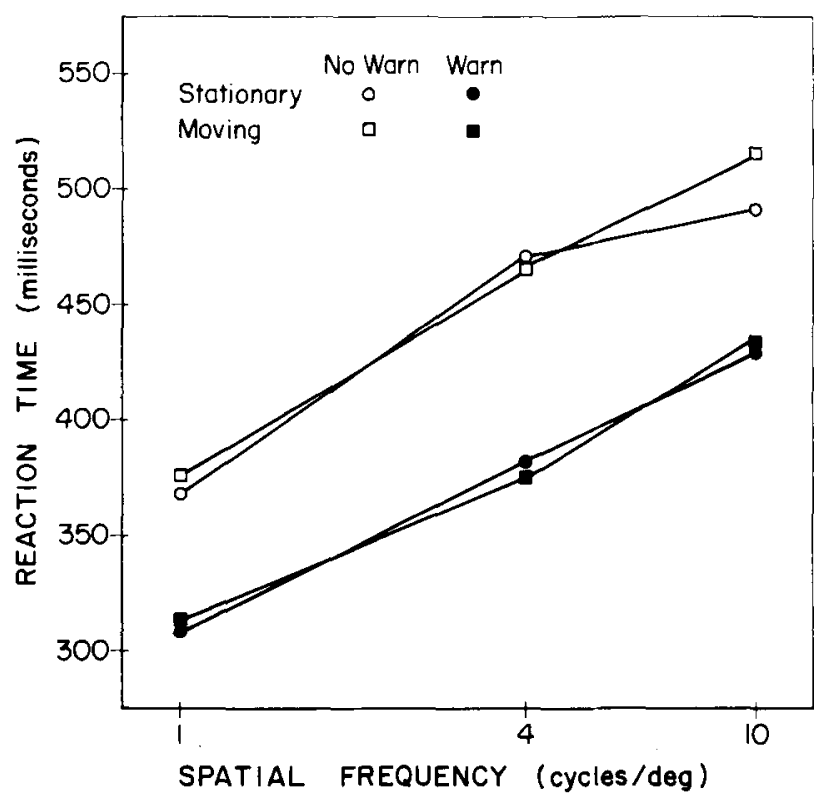

Figure 2. Reaction time (in milliseconds) as a function of spatial frequency with and without warning for stationary and drifting $\left(5^{\circ}\right.$ per second) backgrounds.

The mean false-alarm rates for the functions in Figures 1 and 2, given in Table 1, indicate an increase in mean false-alarm rate in the presence of a warning signal. Also, there were fewer false alarms for the first measurement of threshold than for the second. This was true for both the no-warning and warning conditions, but the change was greater for the warning condition. The analysis of the false-alarm data revealed significant main effects of warning $[F(1,5)=10.23, p<.025]$ and time $[F(1,5)$ $=18.77, p<.01]$ and the interaction of warning and time $[F(1,5)=11.41, p<.025]$. The increase in faisealarm rate in the presence of a warning suggests that subjects traded speed for accuracy (Pachella, 1974).

\section{Discussion}

The presence of a warning signal improved performance for both contrast sensitivity and reaction time. This finding is consistent with predictions based on the effects of temporal uncertainty. However, since the addition of a warning signal also increased false alarms, it may have also affected arousal or alertness. It is likely that both processing efficiency and alertness could account for the warning effect.

Although there was no main effect of background movement, there was a tendency for contrast sensitivity to be lower in the presence of background motion without a warning signal, especially for $1 \mathrm{cpd}$. However, the reaction time data showed no differences at 1 and $4 \mathrm{cpd}$ and only a 25 -msec difference at $10 \mathrm{cpd}$.

The data suggest that subjects were able to perform the threshold task with minimal interference from the background. This is puzzling, in light of the spontaneous reports of most subjects that the background movement 
Table 1

Proportion of False Alarms (Experiment 1)

\begin{tabular}{rcc} 
& No Warning & Warning \\
\hline & Stationary Background \\
$4 \mathrm{cpd}$ & .00 & .02 \\
$10 \mathrm{cpd}$ & .15 & .04 \\
& .00 & .05 \\
$1 \mathrm{cpd}$ & Moving Background \\
$4 \mathrm{cpd}$ & .01 & .07 \\
$10 \mathrm{cpd}$ & .00 & .02 \\
\hline
\end{tabular}

was extremely distracting. In terms of masking, the lack of a background effect could be due to the separation in spatial frequency between the target and the background. This is not surprising, in light of research on spatialfrequency-selective masking effects cited earlier. In terms of eye movements, the results suggest that either the background did not elicit eye movements large enough to affect contrast sensitivity or the measurement was insensitive to fixational instability. The latter possibility was investigated in Experiment 2 by increasing the demands on fixational stability.

\section{EXPERIMENT 2}

The purpose of this experiment was to determine whether sinusoidal background motion, which may be more demanding than the constant-velocity background motion used in Experiment 1, would degrade performance on a grating acuity task requiring high fixational stability.

The reason for using sinusoidal background motion is based on a possible explanation for the absence of a background effect in Experiment 1 . In order to maintain stable fixation in the presence of background motion, pursuit effort is required to oppose the smooth component of the reflex eye movement elicited by background motion (Post \& Leibowitz, 1985). If the background moves at a constant velocity, the required pursuit effort is also constant. However, a sinusoidally moving background would require continual changes in the direction and overall level of pursuit effort to maintain stable fixation and would therefore place higher demands on the pursuit system.

In this experiment the orientation of the target grating was varied to help determine whether fixational instability induced by background motion degraded performance. If vertical background stripes are moved horizontally, the major component of any induced fixation instability would be horizontal. This instability would be expected to produce a greater reduction in retinal image contrast for a vertically oriented than for a horizontally oriented test grating. If there is a background effect for a vertical and not for a horizontal grating, then the effect could be attributed to increased fixational instability. Alternatively, if the background effect is present regardless of orientation, then the effect could be attributed to masking that is not orientation selective (Breitmeyer et al., 1980).

\section{Method}

Subjects. Eight paid subjects, 4 of whom had served in previous experiments, were tested. Each had 20/20 vision or better as determined by a Titmus vision tester.

Apparatus. The sinusoidal background motion was produced by an eccentric cam connected to a variable-speed motor that transformed circular motion of the motor into sinusoidally oscillating linear motion. The cam was connected to the glass cylinder to produce sinusoidal oscillation of the background. The amplitude $\left(20.9^{\circ}\right)$ and frequency $(.25 \mathrm{~Hz}$, or an average velocity of $10.4 \mathrm{dps})$ of background motion were fixed. The background was viewed at $75 \mathrm{~cm}$. The target, although flush with the back surface of the background, was viewed at $75.5 \mathrm{~cm}$ due to the thickness of the glass on the scope. The spatial frequency $(.22 \mathrm{cpd})$ and contrast (.38) of the background were fixed. The spatial frequency of the background was slightly different from that of Experiment 1 because we used a farther viewing distance in this experiment and .22 was the closest we could get to the spatial frequency of Experiment 1 (.16 cpd) using $1 / 16$-in.-wide graphic arts tape. The spatial frequency of the central grating was varied in this experiment to obtain a measure of grating acuity. The contrast of the test grating was constant at $20 \%$. The mean luminance of the central and background gratings was $.7 \mathrm{fL}$. All other conditions were the same as in Experiment 1 .

Procedure. Grating acuity was estimated using a single staircase procedure. When a grating presentation went undetected, the spatial frequency decreased in .06-cpd steps until the subject detected the pattern. A detection response incremented the spatial frequency by $.06 \mathrm{cpd}$ for the next trial. The mean of six yes/no transitions was used as an estimate of grating acuity. However, for the same reasons as in Experiment 1, the staircase thresholds are not reported. Three spatial frequencies ( $20 \%$ contrast) above and below the estimated grating acuity, spaced .02 log unit apart, were chosen for the method-of-constant-stimuli procecture. As in Experiment 1, each block consisted of 80 trials. Grating acuity was determined just as thresholds were determined in Experiment 1. For a given block the background was either moving or stationary and the target was presented with or without warning. Each condition was presented twice within a session and the orientation was varied across sessions. The conditions of this experiment were counterbalanced among subjects. All subjects were given practice prior to the experiment.

\section{Results}

The cell means for the acuity data are presented in Figure 3 for vertical (lower curves) and horizontal (upper curves) orientations. The ordinate is the spatial frequency cutoff (cpd) and the abscissa is warning condition. There was no effect of background motion for the horizontally oriented grating, whereas performance was poorest in the presence of sinusoidal background movement and no warning for the vertical grating. Thresholds were higher in the no-warning condition for both horizontal and vertical gratings. To determine the stability of the thresholds, the first and second measurements of threshold per condition were determined. There were similar increases in threshold for all conditions from first to second measurement (order effect). A four-factor ANOVA (warning $\times$ background $\times$ order $\times$ orientation) performed on the acuity data revealed significant main effects of warning $[F(1,7)=14.69, p<.01]$, background $[F(1,7)=10.62, p<.01]$, and order $[F(1,7)=5.75$, $p<.05]$. The interaction of orientation and background was also significant $[F(1,7)=12.86, p<.01]$. 
The cell means for the reaction time data are plotted in Figure 4 for vertical (lower curves) and horizontal (upper curves) orientations. The reaction times were longer for the stationary background but only when the orientation of the central grating was horizontal. As in Experiment 1 , the presence of a warning signal decreased reac-

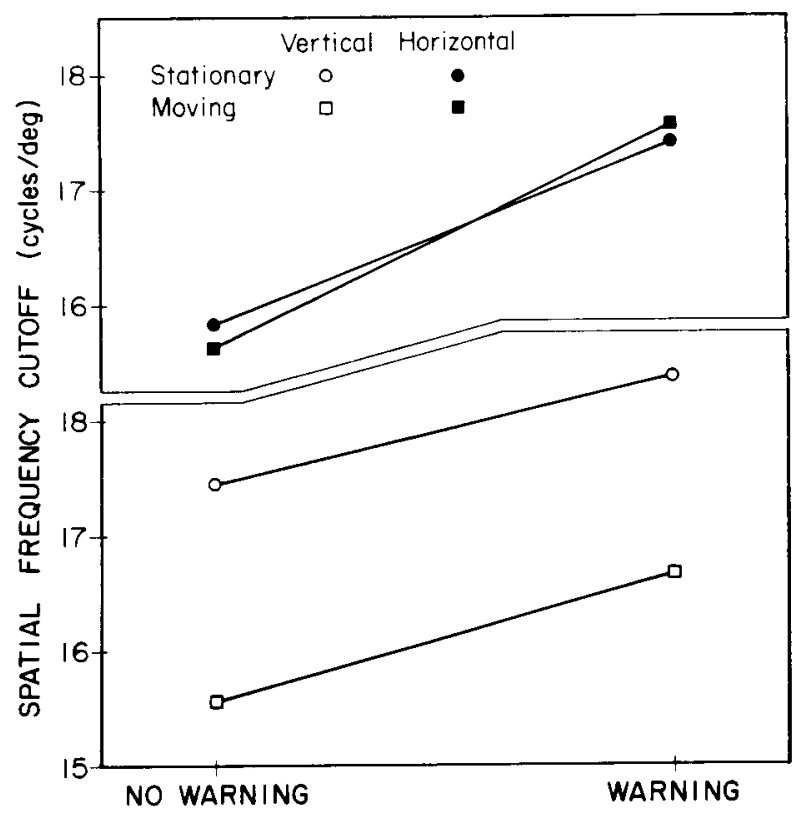

Figure 3. Spatial frequency cutoff with and without warning for vertically and horizontally oriented gratings in the presence of stationary and oscillating $(.25 \mathrm{~Hz})$ backgrounds.

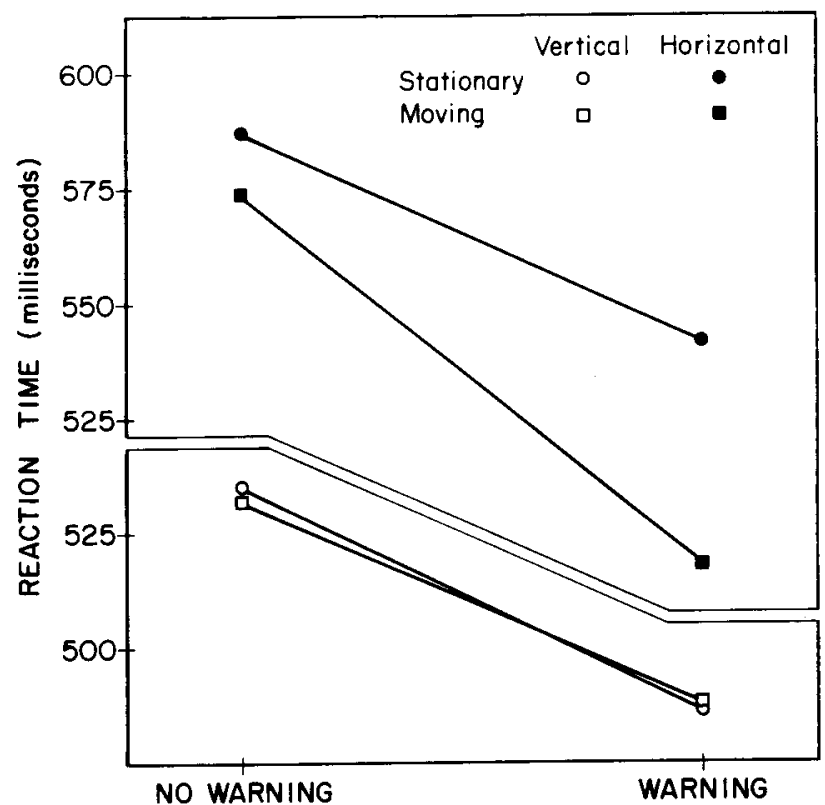

Figure 4. Reaction time with and without warning for vertically and horizontally oriented gratings in the presence of stationary and oscillating (.25 Hz) backgrounds.
Table 2

Proportion of False Alarms (Experiment 2)

\begin{tabular}{lcc} 
& No Warning & Warning \\
\hline Stationary Background & & \\
Horizontal & .01 & .05 \\
Vertical & .00 & .07 \\
Moving Background & & \\
Horizontal & .00 & .07 \\
Vertical & .01 & .09 \\
\hline
\end{tabular}

tion times. The analysis revealed significant main effects of orientation $[F(1,7)=7.02, p<.03]$ and warning $[F(1,7)=59.23, p<.0001]$, and an orientation $\times$ background interaction $[F(1,7)=8.23, p<.02]$.

Mean false-alarm rates for each cell, presented in Table 2 , were not significantly different across warning conditions.

\section{Discussion}

The grating acuity data demonstrate that sinusoidal background movement degraded performance for vertical but not horizontal gratings, suggesting that the effect of the moving background was caused by retinal smear. The presence of a warning signal enhanced overall performance but did not eliminate the detrimental effects of the background movement. A comparison of the results of Experiments 1 and 2 suggests that fixational instability was higher for sinusoidally oscillating backgrounds than for stationary or drifting backgrounds.

For the reaction time data, slower reaction times for horizontally oriented gratings could simply reflect intersession changes. However, of greater interest are the differences in reaction time due to background motion. Reaction times were not different for stationary and moving backgrounds when the central grating was oriented vertically. When the grating was oriented horizontally, performance was better with a moving background. No explanation for this finding is offered.

An alternative explanation for the background effect is based on orientation-specific masking. ${ }^{2}$ The orientationspecific masking hypothesis assumes that performance on the central grating task will be degraded by the moving background when cortical neurons activated by the central and peripheral patterns are tuned to the same orientations (Bowling, 1985a, 1985b; Derrington, 1984). This suggests that the lack of a background effect for the horizontal grating was due to a lack of interference among mechanisms sensitive to different orientations. ${ }^{3}$

In retrospect, it would have been desirable to equate the horizontal and vertical dimensions of the target gratings. The difference between the stationary background conditions for vertically and horizontally oriented targets may well be a result of the decreased number of cycles for horizontally oriented targets. However, the important comparison, for the purpose of evaluating the effect of background movement, was between moving and stationary conditions within an orientation. 


\section{EXPERIMENT 3}

The purpose of this experiment was to assess the effects of background and target movement on a contrast sensitivity task similar to that used in Experiment 1 . In Experiments 1 and 2 only the background was moved. In Experiment 3, to increase demands on the pursuit system, the target was also moved. Pursuit of a moving target against a stationary, patterned background is particularly demanding because it requires the subject to both suppress reflexive eye movements elicited by the background moving across the retina as the target is tracked and pursue the target against patterned backgrounds, which requires the subject to move the eye at a velocity that approximates that of the target so that a stable image of the target is maintained on the fovea.

In Experiment 3 we also attempted to compare changes in contrast sensitivity resulting from fixation instability due to background movement, pursuit inaccuracies, or a combination of these factors. This was accomplished by measuring contrast sensitivity for both stationary and moving targets in the presence of both stationary and moving backgrounds. Decreased contrast sensitivity for a stationary target in the presence of background movement (relative to contrast sensitivity for a stationary target in a stationary background) is largely attributable to fixational instability and/or masking. Decreased contrast sensitivity for a moving target in the presence of a background that moves with the target can be attributed to pursuit inaccuracies, because the background does not move across the retina in this case. When the target moves and the background is stationary, decreases in contrast sensitivity can be attributed to fixation instability and/or masking, as well as to pursuit inaccuracies.

Murphy (1978) showed that pursuit inaccuracies are a limiting factor during pursuit tasks. He reported decreases in contrast thresholds associated with decreases in retinal image speed during pursuit. Also, Collewijn and Tamminga (1984) showed that patterned backgrounds slow down smooth pursuit and increase saccadic eye movements during pursuit. Although they did not measure contrast thresholds, the decreases in gain obtained with structured backgrounds would be expected to decrease contrast sensitivity for high spatial frequencies.

Another factor that may limit performance in dynamic visual tasks is the ability of the observer to perform two tasks simultaneously (Sheehy, 1984). Any factors that affect either the ability to perform two tasks simultaneously or the accuracy of pursuit should affect visual performance during dynamic tasks. Because Experiment 2 showed decreases in grating acuity in the presence of oscillating backgrounds, and because fixation may be considered to be a special case of pursuit at zero target velocity (Robinson, 1981), it was of interest to determine how dynamic visual contrast sensitivity for a high spatial frequency is affected by the presence or absence of background movement. Experiment 3 was designed to determine how patterned backgrounds affect performance on a dynamic con- trast sensitivity task when the background is either stationary or moving with the target.

\section{Method}

Subjects. Eight paid subjects, who had 20/20 visual acuity or better as determined by a Titmus vision tester, were tested.

Apparatus. The display was modified so that the background and target could be moved separately or together. The monitor was mounted on a movable tray so that it could be moved in the moving target condition. As in Experiment 2, rotary motion of the motor was converted to sinusoidally oscillating linear motion by way of a cam. This cam was connected to both the movable tray for the monitor and the glass cylinder for the surround. The linkages connecting the scope and glass cylinder to the cam could be readily disconnected so that the background and target could be moved alone or together.

The target aperture had to be enlarged so that the target gratings could be seen in the moving target conditions in which the monitor moved behind the surface of the background. However, the monitor face was not large enough to cover this hole. A piece of posterboard was mounted onto the movable tray that covered the hole during target motion.

Target presentation was controlled by an Apple Ile computer via magnetic switches that detected target position. The target was presented either at the left or the right extreme of the target excursion or at the center as the target moved to the left or right (to assess any asymmetries in performance for leftward or rightward pursuit). Within a given block of trials the target was presented equally often at a given position and each contrast level was presented nearly the same number of times at each position. Interstimulus intervals of $1,2,3$, and $4 \mathrm{sec}$ were randomly determined. In all other aspects the apparatus was identical to that used in Experiment 2.

Procedure. Threshold was determined for a 12-cpd grating, using the same procedure as in Experiment 1. No warning signal was provided. Subjects were given practice prior to the experimental session.

\section{Results}

Figure 5 presents contrast sensitivities for the two target and two background conditions. The stationary back-

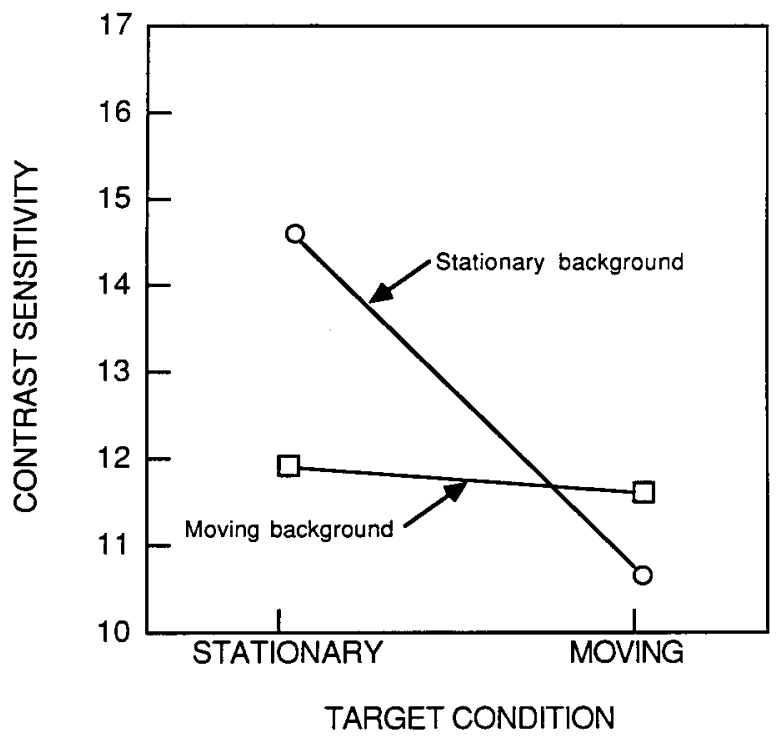

Figure 5. Contrast sensitivity for stationary and moving targets in the presence of stationary and oscillating $(.25 \mathrm{~Hz})$ backgrounds. 
ground and stationary target condition produced the highest contrast sensitivity. Movement of either the target or background degraded contrast sensitivity. A twofactor repeated measures ANOVA (target $\times$ background) on the contrast sensitivity data revealed a significant interaction of target and background $[F(1,7)=60.08$, $p<.00011$. Analyses of reaction time and false-alarm data revealed no significant main effects or interactions.

Since the differences in sensitivity among the four conditions were small, further analyses were performed to determine how robust the relative sensitivities of the four conditions were across target position. The mean contrast sensitivities are plotted in Figure 6 as a function of spatial position for the four combinations of target and background movement. There is no overlap between the contrast sensitivities for stationary background and stationary target, moving background and stationary target, and stationary background and moving target. The relative order of sensitivities is the same as in the previous analysis. However, the moving background and moving target condition overlaps with all but the stationary background and stationary target condition. Also worthy of note is that there are no systematic changes in sensitivity as a function of position. In a three-factor repeated measures ANOVA (target $\times$ background $\times$ position), there was a significant interaction of target and background $[F(1,7)$ $=15.21, p<.01]$. The position analysis suggests that the relative ordering of sensitivities for the four conditions is fairly robust.

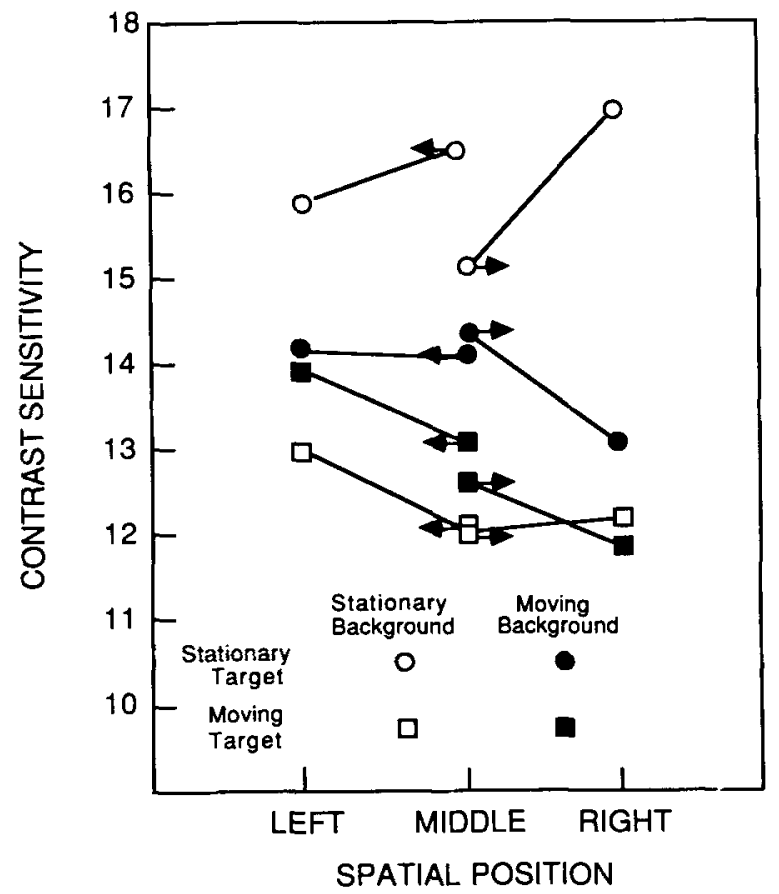

Figure 6. Contrast sensitivity as a function of position. Arrows for data points at the middle position indicate direction of movement.

\section{Discussion}

The results of this experiment indicate that either target or background movement alone decreases contrast sensitivity and that target movement combined with background movement decreases sensitivity somewhat more. These changes are largely attributable to changes in accuracy of fixation and/or pursuit; however, the same conditions may also give rise to masking, since image movement, whether imposed by moving the object or by moving the retina across an image of the object, should cause interference among mechanisms tuned to the same spatial frequencies and orientations. It is unlikely that explanations based on masking can completely explain the results, however, for two reasons: (1) In the moving background and stationary target conditions, the change in speed varied from 0 dps at the endpoints of the excursion to $16.4 \mathrm{dps}$ at the middle. In view of the variability in the velocity of the background, the amount of masking should be greater at the central positions, but this was not the case. (2) In the moving target and moving background condition, there was much less movement of the background on the retina than occurred during the moving background and stationary target condition. Thus, if masking could completely explain the findings, the condition in which both background and target moved together should have a sensitivity closer to that of the stationary background and stationary target condition. In fact, the sensitivity for the moving background and moving target condition was closer to that of the moving background and stationary target condition, suggesting that pursuit inaccuracies, rather than masking, played an important role in the decreased sensitivity when both background and target moved together. ${ }^{4}$ Although the role of masking cannot be ruled out, it cannot completely explain the observed changes in sensitivity. A more parsimonious interpretation is to attribute the present results to inaccuracies of pursuit and fixation.

\section{GENERAL DISCUSSION}

The common issue in the present experiments is performance degradation due to background movement, target movement, and temporal uncertainty. When temporal uncertainty was increased in the absence of a warning signal, performance in Experiments 1 and 2, as assessed by contrast sensitivity, grating acuity, or reaction time, was degraded. The presence of a warning signal to reduce temporal uncertainty resulted in significant decreases in reaction time, increases in contrast sensitivity (Experiment 1), and increases in spatial frequency cutoff (Experiment 2). The improvements are partially attributable to increased alertness in the warning condition, as indicated by the higher rate of false alarms.

Increasing the demands on pursuit effort led to changes in grating acuity (Experiment 2) and contrast sensitivity (Experiment 3). A constant-velocity background move- 
ment did not affect contrast sensitivity (Experiment 1), but when the demands on the pursuit system were increased by a sinusoidal background movement (Experiment 2), performance was degraded. The results of Experiment 3 confirm that sinusoidal background movement decreases contrast sensitivity and that pursuit of a target moving sinusoidally across a stationary background decreases contrast sensitivity somewhat more. These results indicate that increasing pursuit effort demands leads to decrements in performance on a task requiring high fixational stability.

The two factors affecting performance on the tasks used in this series of experiments are temporal uncertainty and background movement. In the absence of information about when the target will occur, observers must sustain their ability to detect targets over longer periods of time. This situation is analogous to many everyday tasks, such as vehicle guidance. However, vehicle guidance also involves many uncertainties in addition to temporal uncertainty that would be expected to degrade performance.

The second factor affecting performance is background movement. In order to maintain performance in the presence of a moving background, observers must exert a pursuit effort to suppress the reflexive eye movements induced by the moving background. The adequacy of this effort will affect performance. This is true for a stationary target viewed against a moving background as well as for a moving target viewed against a stationary background.

In addition to their theoretical relevance, there are practical implications of these data for visual assessment and prediction of performance. Standard assessment techniques are useful in diagnosing visual disorders and suggesting treatment. In many visual tasks encountered outside the laboratory, however, performance is determined, for individuals without gross visual disorders, by how well the observer can meet the demands that are not usually included in standard evaluation procedures. The contribution of variables such as uncertainty and movement, which were investigated in the present series of experiments, must be considered in predicting performance in demanding tasks typically encountered outside the laboratory environment.

\section{REFERENCES}

Bowling, A. (1985a). The effects of peripheral movement and flicker on the detection thresholds of sinusoidal gratings. Perception \& Psychophysics, 37, 181-188.

Bowling, A. (1985b). The effects of the contrast, spatial frequency, and temporal frequency of a surrounding field upon the detection thresholds of gratings. Perception \& Psychophysics, 38, 387-391.

BreITMEYeR, B. G. (1980). Unmasking visual masking: A look at the "why" behind the veil of the "how." Psychological Review, 87, 52-69.

Breitmeyer, B. G., \& VAlberg, A. (1979). Local, foveal inhibitory effects of global, peripheral excitation. Science, 203, 463-465.

Breitmeyer, B. G., Valberg, A., Kurtenbach, W., \& Neumeyer, C. (1980). The lateral effect of oscillation of peripheral luminance gratings on the foveal increment threshold. Vision Research, 20, 799-805.
CAmpbell, F., \& Green, D. (1965). Optical and retinal factors affecting visual resolution. Journal of Physiology (London), 181, 576-593.

Cheng, M., Outeraridge, J. S. (1975). Optokinetic nystagmus during selective retinal stimulation. Experimental Brain Research, 23, 129-139.

CoHN, T. E. (1981). Absolute threshold: Analysis in terms of uncertainty. Joumal of the Optical Society of America, 71, 783-785.

Collewinn, H., TAmminga, E. P. (1984). Human smooth and saccadic eye movements during voluntary pursuit of different target motions on different backgrounds. Joumal of Physiology, 351, 217-250.

DERRINGTON, A. M. (1984). Spatial frequency selectivity of remote pattern masking. Vision Research, 24, 1965-1968.

EARLe, D. C., Lowe, G. (1971). Channel, temporal, and composite uncertainty in the detection and recognition of auditory and visual signals. Perception \& Psychophysics, 9, 177-181.

EIMA, Y., \& TAKAHASHI, S. (1983). Effects of high-contrast peripheral patterns on the detection threshold of sinusoidal targets. Journal of the Optical Society of America, 73, 1695-1700.

GeISLER, W. S. (1984). Physical limits of acuity and hyperacuity. Joumal of the Optical Society of America, 1, 775-782.

GeISLER, W. S., DAvila, K. D. (1985). Ideal discriminators in spatial vision: Two-point stimuli. Joumal of the Optical Society of America, 2, 1483-1497.

GiPPENREITER, Y. B., Romanov, V. Y. (1974). A method of investigation of the internal form of visual acuity. In R. B. MacLeod \& H. L. Pick (Eds.), Perception: Essays in honor of James J. Gibson (pp. 227-249). New York: Cornell University Press.

KELLY, D. H. (1979). Motion and vision: II. Stabilized spatio-temporal threshold surface. Joumal of the Optical Society of America, 69, 1340-1349.

Lasley, D. J., CoHN, T. (1981). Detection of a luminance increment: Effect of temporal uncertainty. Journal of the Optical Society of America, 71, 845-850.

MACKAY, D. M. (1970a). Elevation of visual threshold by displacement of retinal image. Nature, 225, 90-92.

MACKAY, D. M. (1970b). Interocular transfer of suppressive effects of retinal image displacement. Nature, 225, 872-873.

Marrocco, R. T., Carpenter, M. A., \& Wright, S. E. (1985). Spatial contrast sensitivity: Effects of peripheral field stimulation during monocular and dichoptic viewing. Vision Research, 25, 917-924.

McIlwain, J. T. (1964). Receptive fields of optic tract axons and lateral geniculate cells: Peripheral extent and barbiturate sensitivity. Journal of Neurophysiology, 27, 1154-1173.

McIlwaIN, J. T. (1966). Some evidence concerning the physiological basis of the periphery effect in cat's retina. Experimental Brain Research, 1, 265-271.

MURPHY, B. J. (1978). Pattern thresholds for moving and stationary gratings during smooth eye movements, Vision Research, 18, 521-530.

Pachella, R. G. (1974). The interpretation of reaction time in information-processing research. In B. H. Kantowitz (Ed.), Human information processing: Tutorials in performance and cognition. Potomac, MD: Erlbaum.

Post, R. B., Leibowitz, H. W. (1985). A revised analysis of the role of efference in motion perception. Perception, 14, 631-643.

Robinson, D. A. (1981). Control of eye movements. In V. B. Brooks (Ed.), Handbook of physiology: Vol. 2, Pt. 2. The nervous system (pp. 1275-1320). Baltimore: Williams \& Wilkins.

SHEEHY, J. B. (1984). Dynamic resolution. Unpublished doctoral dissertation, The Pennsylvania State University, University Park.

VALBERG, A., \& BREITMEYer, B. (1980). The lateral effect of oscillation of peripheral luminance gratings: Test of various hypotheses. $V i$ sion Research, $20,789-798$.

WERTHEIM, A. (1980). Information processed in ocular pursuit. In G. E. Stelmach \& J. Requin (Eds.), Tutorials in motor behavior. New York: North-Holland.

\section{NOTES}

1. Ejima and Takahashi (1983) showed that thresholds for a range of spatial frequencies in the presence of a stationary background mask 
do not decrease when there are more than five cycles in the pattern. Given that the horizontal extent of the aperture was $5.8^{\circ}$, there were $5.8,23.2$, and 58 cycles in the 1-, 4-, and 10-cpd gratings used in this experiment. Therefore, it is unlikely that threshold differences between these spatial frequencies could be attributed to an insufficient number of cycles.

2. The authors are grateful to Boris Crassini for this suggestion.

3. Two comments about masking are worthy of note. First, unpublished data from this lab suggest that constant-velocity background movement has no effect on grating acuity under the same viewing conditions as those in Experiment 2. This finding is difficult to explain using an orientation-specific masking hypothesis, since the backgrounds in both experiments should have activated mechanisms tuned to the same orientations. Second, the "jerk" effect postulated by Breitmeyer et al. (1980) does not involve orientation specificity, since this psychophysical effect is presumed to be a manifestation of neural activity in the retina and lateral geniculate nucleus (Mcllwain, 1964, 1966), where orientationselective cells do not exist. Therefore, it seems likely that orientationspecific masking and the jerk effect are manifestations of interference at different levels in the visual system.

4. The sensitivity for the moving background and moving target condition is slightly higher than that for the stationary background and moving target condition. Although the difference is not significant, it suggests that when the background moved with the target it aided pursuit.

(Manuscript received August 25, 1986; revision accepted for publication January 5, 1988.) 